\title{
THE INFLUENCE OF BASIC LEAD ACETATE ON THE OPTICAL ROTATION OF SUCROSE IN WATER SOLUTION.
}

\author{
By Frederick Bates and J. C. Blake.
}

In the polarimetric estimation of sucrose in raw sugars an indefinite amount of basic lead acetate in solution is usually added to the sugar flask before making up to volume in order to precipitate the impurities more or less completely and thus clarify the sugar solution sufficiently for reading on the polariscope. The use of this reagent is almost universal, and it is so powerful a clarifying reagent that the question whether it can be used in precision work on raw sugars is of the first importance. Except for the error due to the volume of the precipitate which is formed by the reaction it is usually regarded by commercial chemists as giving accurate results.

The evidence obtainable from the literature of the subject as to the effect of basic lead acetate on the polarization of sucrose is conflicting and very unsatisfactory, the results being usually expressed in tenths of a per cent with no indication that attention was paid to numerous minor sources of error. Von Lippmann's ${ }^{1}$ conclusion, drawn from the results of other investigators, is that basic lead acetate exerts no influence on the rotation of sucrose in water solution. We have found this conclusion to be in error. Its great influence on the specific rotation of levulose ${ }^{2}$ has long been known. Nevertheless, because of the relatively large anount of sucrose present in raw sugars as compared with the anount of levulose present, the effect of the basic lead acetate on the sucrose, though relatively less than its effect on levulose, still retains an equal importance.

The effect of basic lead acetate on the specific rotation of sucrose was overlooked by the early investigators, because of the comparatively crude polarizing apparatus and methods used, and also because 
the temperature coefficient of pure sugar solutions was little known or entirely neglected. A few preliminary experiments soon demonstrated that it wonld not be safe to carry ont the present investigation on an ordinary commercial saccharimeter alone. An accuracy of 0 .02 Ventzke was desired. The greatest inherent difficulty in obtaining this accuracy lay in the polarizing apparatus. It was overcome by improving a Josef and Jan Frič donble quartz wedge compensation saccharimeter. The instrument has glass scales and verniers, the light reaching the eye of the observer being transmitted and not reflected. As a result, the effect of temperature changes on the length of the scale is very small, and the definition of the ruling is such that the scale conld readily be interpolated to hundredths of a degree Ventzke. The polarizing nicol was removed anid a firstclass Lippich half-shade system substituted. The light was passed through $\mathrm{I} .5 \mathrm{~cm}$ of a 6 per cent potassinm bichromate solution before it entered the polarizing system. An improved electric lamp of great intensity was used as the source of light, thereby permitting the use of a polarizing angle of $4^{\circ} \cdot 33$ (circular). This angle is too small to be utilized with the ordinary mechanisin for shifting the wedge. The instrument was accordingly fitted with an anxiliary screw adjustment by means of which the accurate settings were made. With the improvements as indicated above the sensitiveness of the saccharimeter was such that a difference in rotation of 0.02 Ventzke conld be detected with certainty by the observer, withont nnaking a series of observations, differences of that magnitude being sufficient to produce a directly discernible change in the intensities of the two halves of the field. In Table I are given ten consecntive readings on a solution in this instrument. They were selected at random.

\section{TABLE I.}

Consecutive Readings on Sugar Solution.

(In $200 \mathrm{~mm}$ tube.)

\begin{tabular}{|c|c|c|}
\hline Degrees Ventzke & Degrees Ventzke & Degrees Ventzke \\
\hline $99 ? 98$ & $99: 99$ & $99: 98$ \\
\hline 100.00 & $99: 99$ & $99: 98$ \\
\hline $99: 99$ & $99: 98$ & $99: 98$ \\
\hline $99: 99$ & & \\
\hline
\end{tabular}


The surfaces of the wedges were sufficiently plane to justify this degree of refinement. The length used where the differences in rotation were measured corresponded to not more than I? I V of the scale. The Ioo cc flasks were especially made for this work. The necks had an internal diameter of ro $\mathrm{mm}$. The volumes were known to within O.OI cc. All temperatures were read on thermometers graduated to 0 . I. The tubes were $200 \mathrm{~mm}$ in length and enlarged at one end. A glass tube $2.5 \mathrm{~cm}$ long projected from the middle of the tube and at right angles to its length. Through this side tube a thermometer was passed, its bulb being immersed in the solution. A rubber washer closed the space between the tube and thermometer. All the apparatus used was standardized for a temperature of $20^{\circ}$.

The sugar used in these experiments was the best commercial grade. Two samples were carefully taken from sugar purchased at different times. Each sample was thoroughly mixed. After much consideration it was concluded that practically nothing would be gained by using chemically pure sucrose. The large amount required would have been a serious difficulty. The basic lead acetate solution was prepared either by the action of the neutral acetate in solution on lead oxide at the boiling temperature or by simply dissolving a high grade product in water. The solution was made up to a density of $\mathrm{I} .25$ at $\mathrm{I} 5^{\circ}$, and when titrated with acid gave approximately the theoretical amount of dissolved lead oxide.

Investigations of this character should be carried ont in a room whose temperature can be maintained at the standard temperature of $20^{\circ}$. Such a room was not available at the time these experiments were made, but the results were carefully corrected for temperature changes, although these corrections were very small. The temperature coefficient of a pure sucrose solution containing 26 grams of sucrose in roo true cc when made up and polarized at a temperature other than the standard temperature of the apparatus has been redetermined in this laboratory for both a Schmidt and Haensch polariscope and the improved Fric instrument described above. From these results, not yet published, and the fairly concordant results previously obtained by others on the Schmidt and Haensch instrument, the following values were adopted for makingtemperature corrections for variations from $20^{\circ}$ not exceeding $5^{\circ}$ (except in two instances): 
I. Temperature coefficient due to cubical expansion of standard sugar solution at $2.3^{\circ} \pm 2^{\circ}=0^{\circ} .029 \mathrm{~V}$.

2. Temperature coefficient of standard sugar solution made up at $23^{\circ} \pm 2^{\circ}$ read with glass tubes on a quartz wedge polariscope at $23^{\circ} \pm 2^{\circ}=0.03 \mathrm{IV}$.

Although the coefficient of expansion of a standard sugar solution varies with the temperature, yet the value here given is sufficiently accurate for our purpose since the difference between the temperature of filling the flasks and the polarizing temperature was always less than $2^{\circ}$. Hence the error could hardly reach 0 . oI $\mathrm{V}$.

The method of procedure finally adopted was as follows:

(I) $26.0480 \pm 0.0003$ grams of sugar were weighed ont, all the weighings for one day being made within a short interval of time. The error due to weighing was, therefore, \pm 0 :oor $\mathrm{V}$.

(2) The sugar was washed into a freshly cleaned flask with ordinary distilled water and dissolved.

(3) The basic lead acetate solution was then added, usually resulting in a turbidity. This turbidity is especially troublesome for amounts of basic lead acetate solution in the neighborhood of 3 to $4 \mathrm{cc}$. It can not be filtered ont with paper filters.

(4) One or two cc of a lead sulphate crean of constant composition were then added to the solntion to facilitate filtration.

(5) The solution was then made up to the mark by inmersing the flask in a water bath, the temperature of filling being determined by means of another flask filled with water similarly placed. The time thus required insured the complete drainage of the water in the neck of the flask. The solution was made as nearly homogeneous as possible before filling, to avoid errors due to contraction or dilution.

(6) The solution was then thoroughly shaken up and poured into a barium sulphate "Faltenfilter" (Schleicher and Schüll No. 605). The filtrate was usually clear at the start. The portion used for polariscopic reading was taken after the liquid had been on the filter almost one minute.

(7) The polariscopic tubes were filled by simple pouring and the solutions polarized on the apparatus described above. The readings were made in a room $(\mathrm{I} .4 \mathrm{~m} \times 2$. I $\mathrm{m} \times 4 \mathrm{~m})$ especially constructed for work of this nature. It was made by partitioning off one corner of the laboratory, and permitted the exclusion of all extraneous light. 
It was maintained at practically the same temperature as the laboratory, by means of air circulation controlled by an electric fan. The temperature corrections were applied as follows.

The average of the temperature of the solution and the temperature read on a thermometer hanging with its bulb in contact with the polariscope was taken as the polarizing temperature. The difference between this temperature and $20^{\circ}$ added to the difference between the polarizing temperature and the temperature of filling (which was always the smaller value) was multiplied by 0.03 and added to the polariscopic reading. The volume of the lead sulphate added was then subtracted and the corrections for the flasks and polariscopic tubes at $20^{\circ}$ applied.

The lead sulphate added to facilitate filtration had no effect on the polariscopic reading after allowance was made for the volume of the sulphate. This is shown by the data in Table II, which contains six separate determinations, each in duplicate, three of which contained $2 \mathrm{cc}$ of lead sulphate and three contained no lead sulphate. The volume of the sulphate in I cc of the cream was $0.045 \mathrm{cc}$. After the data given in Table III had been obtained it was found that the turbidity could be avoided by using water free from carbonates and preventing too great a dilution of portions of the lead subacetate stigar solution in filling the flask to the mark. Accordingly, much of the work was repeated without the use of any lead sulphate. The results were in complete agreement with those given in Table III.

TABLE II.

Effect of $2 \mathrm{cc} \mathrm{PbSO}_{4}$.

\begin{tabular}{|c|c|c|c|c|c|}
\hline \multicolumn{3}{|c|}{ With $\mathrm{PbSO}_{4}$} & \multicolumn{3}{|c|}{ Without $\mathrm{PbSO}_{4}$. } \\
\hline Date & Bates & Blake & Date & Bates & Blake \\
\hline May 2. & $99: 90$ & $99: 89$ & Apr. 28. & $99: 90$ & $99: 92$ \\
\hline June 12 & $99: 895$ & $99: 90$ & May 3. & $99: 895$ & $99: 89$ \\
\hline June 28 & $99: 895$ & $99: 895$ & June 9. & $99: 920$ & $99: 895$ \\
\hline
\end{tabular}

Average without $\mathrm{PbSO}_{4}$ Average with $\mathrm{PbSO}_{4}$ Difference
$99: 903$

$99: 896$ 
The temperature coefficient of the normal sugar solution is not changed appreciably by the addition of basic lead acetate; the coefficient for a pure sugar solution is therefore used. This was deternined by measuring the temperature coefficient of a normal sugar solution containing $20 \mathrm{cc}$ of the basic lead acetate solution. The range of temperature was $\mathrm{I} 4^{\circ}$. The result obtained was in complete agreement with the value given by Schönrock, who found ${ }^{1}$ for the normal sugar solution

$$
a_{20}=a_{\mathrm{t}}+a_{\mathrm{t}}(0.00046 \mathrm{I})(t-20)
$$

for all wave lengths. The coefficient $0.046 \mathrm{I}(0.00046 \mathrm{I} \times \mathrm{IOO}=0.046 \mathrm{I})$ was measured in preference to the coefficient $0.03 \mathrm{I}$, because, while both involve the temperature coefficient of the specific rotation, which alone would be appreciably affected by the presence of basic lead acetate, the $0.03 \mathrm{I}$ involves the temperature coefficient of the instrument and 0.046I does not.

The data on the measurements is given in Table III. The first and second colnmns give the dates on which the different polarizations were made. The third column contains the number of cc of basic lead acetate added. The differences between the solution containing no basic lead acetate and one containing a given amount is given for both observers in the columns headed Sample No. I and Sample No. 2. The + sign indicates that the solution containing the reagent gives the higher polarization, and conversely for the sign. The average of the differences found by both observers is given in the final column. At least five settings were made by each observer on each tube. Of the large number of observations made only three isolated ones, which were obviously in error, have been discarded. Each difference given in the table is the arerage of two separate determinations made sinnltaneonsly except when otherwise stated.

The results seem to be consistent within the linits of accuracy sought $(0.02 \mathrm{~V})$. Eiven when considerable variations occurred, as with 2.5 cc basic lead acetate acting on sugar No. 2, the average value fell pretty close to the smooth curve plotted in Fig. I. The constancy of the blank determinations made on sugar No. 2 was remarkable. 'The value of sugar No. I, on the contrary', continually 
decreased, the total change from March 23 to May 25 being O ${ }^{\circ} 5 \mathrm{~V}$. The value of sugar No. 2 was, therefore, assumed constant for days on which no blank determination was made; whereas values obtained by interpolation were used under similar circumstances for sugar No. I. The changes in No. I were so gradual and extended over such a long time interval that no error larger than $\mathrm{O}$. or $\mathrm{V}$ could have been introduced by the interpolation. The variations between the two observers are of opposite sign on the blank determinations of the two sugars, and are therefore accidental. The data are plotted graphically in Fig. I. Owing to the length of the curve it is plotted in three sections, all on the same scale. The ordinates, in degrees Ventzke, are the differences between the polarizations of two solutions, one of which contains basic lead acetate, the other none. The zero point represents the blank solution. When the polarization given by the basic lead acetate solution is the larger, the difference is given the + sign, and inversely for the - sign. The abscissas are the volume of basic lead acetate added expressed in cc.

The curve shows beyond doubt that under the given circumstances basic lead acetate first causes a lowering of the polariscopic reading of sugar in solution amounting to more than 0 : I $\mathrm{V}$ for normal concentration, and that further addition of the same reagent causes a continuous rise in the polarization up to the limit $(63 \mathrm{cc})$ investigated. It will be observed that when about $6 \mathrm{cc}$ of lead subacetate are added the polarization is not affected, the curve crossing the axis at this point.

This effect of basic lead acetate on the polarization of sugar seems to be due to the formation of soluble lead saccharates having specific rotations different from that of sugar, 'lead saccharates having properties which would account for the general nature of the curve having already been studied in connection with this investigation.

The elevation of the curve shows clearly the error that may be introduced in polarizations where relatively large amounts of the basic lead acetate are used. The depression in the curve, corresponding to small amounts of the reagent, is of special significance in the polarimetric estimation of sucrose in raw sugars, and is of sufficient magnitude to class it with the errors introduced by the volume of the precipitate, the temperature coefficient, and the presence of invert sugar and other impurities.

$2226 \mathrm{I}-\mathrm{O} 7-\mathrm{S}$ 


\section{TABLE III.}

Effect of Basic Lead Subacetate on Normal Solutions.

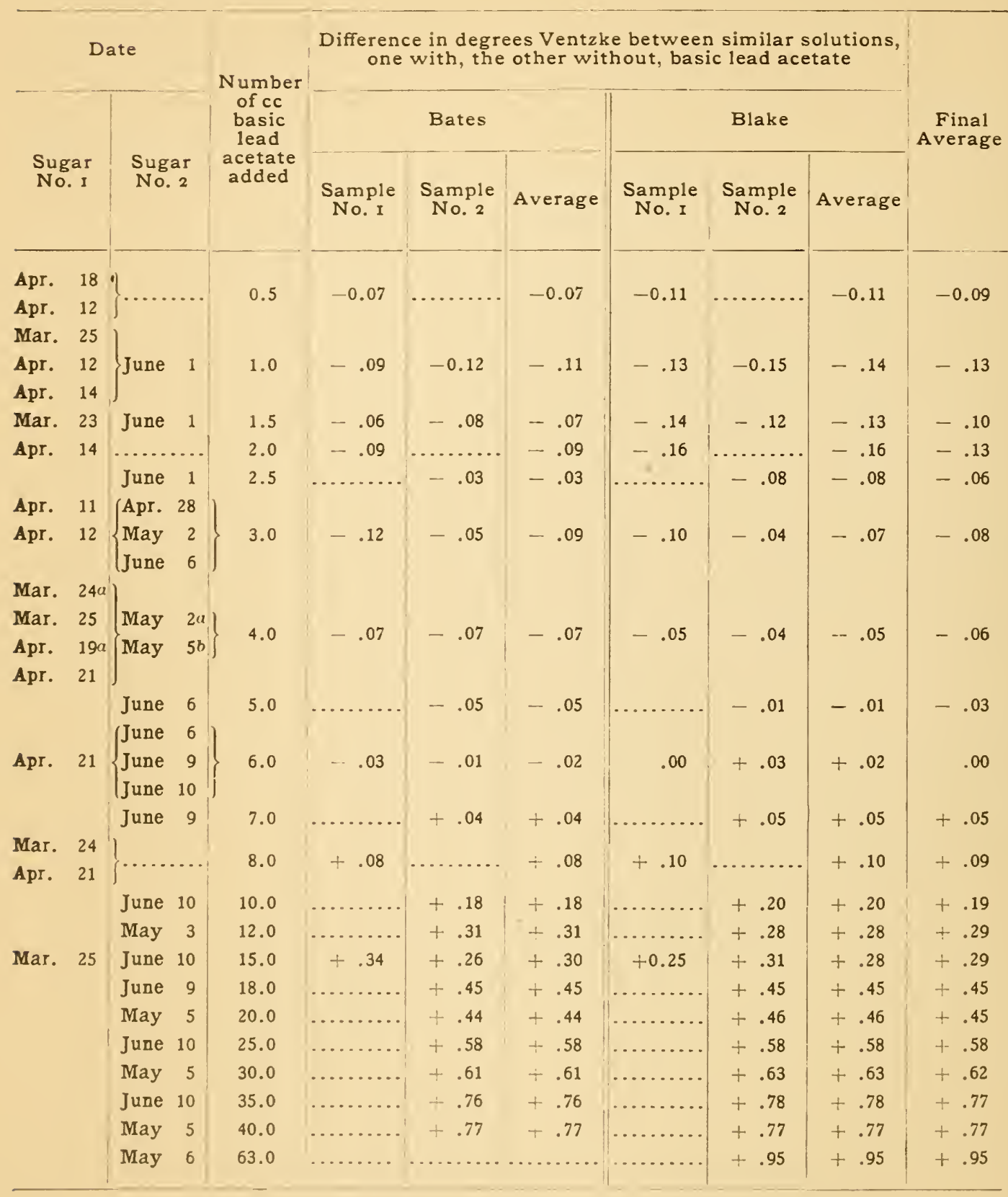




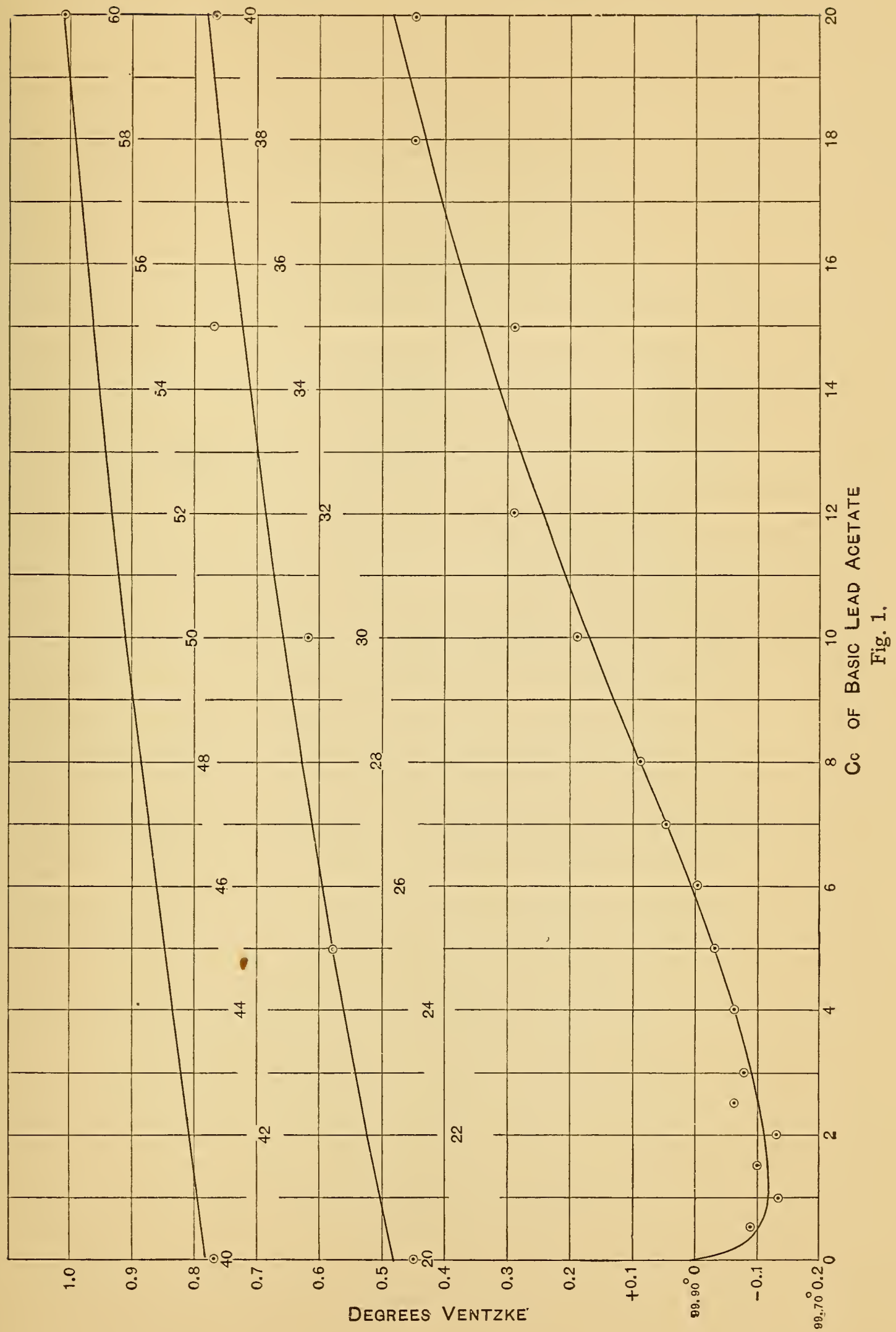

